\title{
ARITHMETIC OF ORTHOGONAL GROUPS (II)
}

\author{
TAKASHI ONO
}

In [0], ${ }^{1)}$ the writer proved some theorems of Hasse type for two orthogonal groups which operate on the same vector space. In this paper, we shall further generalize those results in two directions. One is to consider the propositions of that type for two orthogonal groups which operate respectively on two vector spaces whose dimensions are different from each other, and the other is to deal with some conspicuous subgroups of an orthogonal group simultaneously which play important roles in the structure theory for orthogonal groups. For this reason, the present paper consists of three steps $\S 1, \S 2$ and $\S 3$ which give the generalizations in the above sense of the results in the corresponding sections of $[0]$.

$\S 1$ is of purely algebraic nature and the ground field $K$ may be an arbitrary field of characteristic $\neq 2$. The fundamental statement (Theorem 1 ) shows that the possibility of imbedding (in a certain sense) a group in another group is equivalent to the representability (in a certain sense) of a form by another form each of which corresponds to one of those groups respectively. In $\S 2$, we investigate, in the case where $K$ is a locally compactly valued field, local properties of the groups and forms. These are summarized in Theorem 2 . It is remarkable that though the Clifford algebras are used explicitely to characterize the usual representability of forms over our $K$, the imbedding of groups, which is the same thing as the similar representability of forms, may be characterized by the indices of forms which are a much simpler notion than the Clifford algebras. Lastly, in $\S 3$ where we assume that $K$ is a field of algebraic numbers or a field of algebraic functions of one variable over a finite field of characteristic $\neq 2$, we first prove theorems of Hasse type for the representabilities of forms (Theorem 3,4 ), which are essentially reduced to the fundamental theorem of algebras over our $K$. Then, we may immediately transfer Theorem 4 to that for groups (Theorem 7). Here, we observe some examples of the

Received May 19, 1955.

1) Numbers in brackets refer to the References at the end of the paper. 
fact that 'a property $I I$ of an object $O(K)$ follows from the fact that the object $O_{\mathfrak{p}}(K)$, which is obtained naturally from $O(K)$ by transition to $K_{\mathfrak{p}}$ from $K$, has a weaker property $\Pi^{\prime}$ than $\Pi$ for every place $p$ in $K^{\prime}$ (Theorem 6,8 etc.). It seems interesting to the writer that such facts are, in the present case, based on Theorem 5 which is stated by making use of the indices of forms.

The writer wishes to express his thanks to Mr. T. Tamagawa who gave him a suggestion on the arithmetic of algebras.

\section{$\S 1$. Preliminaries}

Let $K$ be a field of characteristic $\neq 2$, and let $V$ and $W$ be finite dimensional vector spaces over $K$. Suppose that there is a semi-linear mapping $\Theta$ of $W$ into $V$. Let $f$ be a symmetric bilinear form on $V$. We denote by $\Theta f$ a form on $W$ which is defined as follows:

$$
\Theta f(x, y)=(f(\Theta x, \Theta y))^{\theta^{-1}} \text { for } x, y \in W,
$$

where $\theta$ is the automorphism of $K$ associated with $\Theta$. It is easily verified that thus obtained $\Theta f$ is again symmetric bilinear. Now, let $\Phi$ be another semi-linear mapping of the third space $U$ into $W$ with the associated automorphism $\varphi$ of $K$, then the mapping $\Theta \circ \emptyset$ of $U$ into $V$ is a semi-linear mapping with the automorphism $\varphi \theta$, and it follows at once that $(\Theta \circ \emptyset) f=\emptyset(\Theta f)$ for the form $f$ on $V$. Particularly if $\Theta$ is a semi-linear isomorphism between $W$ and $V$, then we have $d(\Theta f) \sim d(f)^{\theta^{-1}}$ and $\nu(\Theta f)=\nu(f)$ for a nondegenerate form $f$ on $V$, where $d(f)$ is the discriminant of the form $f^{2)}$ relative to some basis of $V$ and $\nu(f)$ is the index of $f^{3}{ }^{3}$

Now suppose that two nondegenerate forms $f$ and $g$ are given on the spaces $V$ and $W$ respectively. We say that $f$ semi-similarly represents $g: f \stackrel{\leftrightarrow}{\rightarrow}$, if $g=\lambda \cdot \Theta f$ for some semi-linear injection $\Theta$ of $W$ into $V$ and for some $\lambda \in K^{*}$. $^{\text {) }}$ If the injection $\Theta$ is linear, we say that $f$ similarly represents $g: f \stackrel{\infty}{\rightarrow} g$, and, furthermore, if the scalar $\lambda=1$, then we say simply that $f$ represents $g: f \widetilde{g}$. Particularly if $V$ and $W$ have the same dimensionalities, we take away the arrows in the above notations and say that $f$ and $g$ are semi-similar : $f \circ g$, similar : $f \infty g$ and congruent $: f \sim g$ respectively.

2) We often use simply the word 'form' for 'nondegenerate symmetric bilinear form.'

3) See the footnotes 15) in [0].

4) Semi-linear injection of $W$ into $V$ means the isomorphism of $W$ into $V . K^{*}$ is the set of all non-zero elements in $K$. 
Proposition 1. Let $f$ and $g$ be nondegenerate symmetric bilinear forms on the spaces $V$ and $W$ respectively. Then, in order to have $f \stackrel{\leftrightarrow}{\rightarrow}(f \stackrel{\infty}{\rightarrow} g$ or $f \leadsto g)$ it is necessary and sufficient that there exists a form $h$ on a suitable space $U$ such that $f \mathrm{co} g+h(f \infty g+h \text { or } f \sim g+h)^{5}{ }^{5}$

Proof. Suppose that there exists a form $h$ on a space $U$ such that $f \propto s g+h$. Then, there is a semi-linear isomorphism $\Theta$ between $W \times U$ and $V$ and an element $\lambda \in K^{*}$ such that $g+h=\lambda \cdot \Theta f$. Let $\Theta^{\prime}$ be the restriction of $\Theta$ to $W\left(\Theta^{\prime}(w)\right.$ $=\Theta(w, 0), w \in W)$. Then, $\Theta^{\prime}$ is clearly a semi-linear injection of $W$ into $V$ such that $g=\lambda \cdot \Theta^{\prime} f$. Thus, we have $f \stackrel{\leftrightarrow}{\rightarrow} g$. Conversely, suppose that $f \stackrel{\leftrightarrow}{\rightarrow} g$. Then, there is a semi-linear injection $\Theta^{\prime}$ of $W$ into $V$ and an element $\lambda \in K^{*}$ such that $g=\lambda \cdot \Theta^{\prime} f$. It is clear that the image space $\Theta^{\prime}(W)$ is non-isotropic in $V$. Therefore we get an orthogonal decomposition, $V=\Theta^{\prime}(W) \oplus U$. Let $\Theta^{\prime \prime}$ be the semi-linear automorphism of $U$ with the same associated automorphism as $\Theta^{\prime}$. Using this $\Theta^{\prime \prime}$, we put $h=\lambda \cdot \Theta^{\prime \prime} f$ on $V$ and define a mapping $\Theta$ of $W \times U$ into $V$ by putting $\Theta(w, u)=\Theta^{\prime}(w)+\Theta^{\prime \prime}(u), w \in W, u \in U$. Then, it follows immediately that $\Theta$ is a semi-linear isomorphism between $W \times U$ and $V$ and $\lambda \cdot \Theta f=g+h$. Thus, we get $f \sim s g+h$. By specializing the conditions on $\Theta$ and $\lambda$, we get at once the propositions for the similar representability and the representability of forms.

Proposition 2. Let $f$ and $g$ be forms on the spaces $V$ and $W$ respectively. If $f$ semi-similarly represents $g: f \stackrel{\circ}{\rightarrow} g$, then, we have $0 \leqq \nu(f)-\nu(g) \leqq \operatorname{dim} V$ $-\operatorname{dim} W$.

Proof. Let $\Theta$ be the semi-linear injection of $W$ into $V$ such that $g=\lambda \cdot \Theta f$, $\lambda \in K^{*}$. Then, $\Theta(W)$ is non-isotropic in $V$ and we have $g=\lambda \cdot \Theta f_{1}$, where $f_{1}$ is the restriction of $f$ to $\Theta(W)$. Since $g \propto s f_{1}$, we have $\nu(g)=\nu\left(f_{1}\right) \leqq \nu(f)$. This proves the first inequality. To prove the second inequality, it is sufficient to show that $\nu(f)-\nu\left(f_{1}\right) \leqq \operatorname{dim} V-\operatorname{dim} W_{1}, W_{1}=\Theta(W)$. Let $V=W_{1} \oplus W_{2}$ (orthogonal decomposition) and let $f_{2}$ be the restriction of $f$ to $W_{2}$. First, assume that $\nu\left(f_{1}\right)=0$, and suppose that $\nu(f)>\operatorname{dim} W_{2}$. Let $U \subset V$ be a totally isotropic subspace of dimension $\nu(f)$, and $u_{i}(i=1, \ldots, \nu(f))$ be its some basis. According to the above orthogonal decomposition, we set $u_{i}=w_{i}^{1}+w_{i}^{2}, w_{i}^{1} \in W_{1}$,

5) $g+h$ is the form on $W \times U$ defined by $(g+h)\left(\left(w_{1}, \boldsymbol{u}_{1}\right),\left(w_{2}, \boldsymbol{u}_{2}\right)\right)=g\left(w_{1}, w_{2}\right)+h\left(\boldsymbol{u}_{1}, \boldsymbol{u}_{2}\right)$ for $w_{i} \in W, u_{i} \in U i=1,2$. 
$w_{i}^{2} \in W_{2}, i=1, \ldots, \nu(f)$. Since $\nu(f)>\operatorname{dim} W_{2}$, there is a nontrivial linear relation $\sum_{i=1}^{\nu(f)} \lambda_{i} w_{i}^{2}=0$. On the other hand, as $u_{i}$ is a basis of $U$, we get $u=\sum \lambda_{i} u_{i}$ $=\sum \lambda_{i} w_{i}^{1} \neq 0 \in W_{1}$. Since $u \in U$, it follows that $f_{1}(u, u)=0$. This contradicts the assumption that $\nu\left(f_{1}\right)=0$. Next, suppose that $\nu\left(f_{1}\right) \neq 0$, and let $W_{1}=W_{1}^{\prime} \oplus W_{1}^{\prime \prime}$ be the orthogonal decomposition of $W_{1}$ such that $\nu\left(f_{1}\right)=\nu\left(f_{1}^{\prime}\right), \nu\left(f_{1}^{\prime \prime}\right)=0$, where $f_{1}^{\prime}$ and $f_{1}^{\prime \prime}$ are the restrictions of $f$ to $W_{1}^{\prime}$ and $W_{1}^{\prime \prime}$ respectively. Applying the first case to the space $W_{1}^{\prime \prime} \oplus W_{2}$, we have $\nu\left(f_{1}^{\prime \prime}+f_{2}\right) \leqq \operatorname{dim} W_{2}$. Since $\nu(f)$ $=\nu\left(f_{1}^{\prime}\right)+\nu\left(f_{1}^{\prime \prime}+f_{2}\right)=\nu\left(f_{1}\right)+\nu\left(f_{1}^{\prime \prime}+f_{2}\right)$, we also have the second inequality for this case.

Now, for a form $f$ on a space $V$, we denote by $O(V, f)$ the orthogonal group of $f$, by $O^{+}(V, f)$ the rotation group of $f$ and by $\Omega(V, f)$ the commutator subgroup of $O(V, f)$. Furthermore, we denote by $\Gamma(V, f)$ any one of these three types of groups. It is then clear that $\Gamma(V, \lambda f)=\Gamma(V, f)$ for any $\lambda \in K^{*}$. Let $W$ be another vector space and $\Theta$ be a semi-linear isomorphism between $W$ and $V$. Let $\sigma$ be a linear automorphism of $V$. We define a linear automorphism $\Theta_{\sigma}$ of $W$ by putting

$$
\Theta \sigma(w)=\Theta^{-1} \sigma \Theta(w), \quad w \in W .
$$

It is easy to see that $\Theta \sigma \tau=\Theta \sigma \cdot \Theta \tau$ and $\sigma$ leaves $f$ invariant if and only if $\Theta_{\sigma}$ leaves $\Theta f$ invariant. Moreover, observing that the group $\Omega(V, f)$ is generated by the squares of the elements in $O(V, f){ }^{6)}$ we have $\Theta(\Gamma(V, f))=\Gamma(W, \Theta f)$, where the groups $\Gamma$ 's on both sides belong to the same type of that three groups." Assume that there are forms $f, g$ on the spaces $V, W$ respectively such that $f \stackrel{\leftrightarrow}{\rightarrow} g$. By definition there exists a semi-linear injection $\Theta$ of $W$ into $V$ such that $g=\lambda \cdot \Theta f$ for some $\lambda \in K^{*}$. Applying the above consideration to the form $f_{1}$ on $\Theta(W)$ which is the restriction of $f$ to $\Theta(W)$, we get $\Theta\left(\Gamma\left(\Theta(W), f_{1}\right)\right)$ $=\Gamma(W, g)$. Thus, we say that the group $\Gamma(W, g)$ is semi-linearly imbedded in $\Gamma(V, f)$ if

$$
\Theta\left(\Gamma\left(\Theta(W), f_{1}\right)\right)=\Gamma(W, g)
$$

for some semi-linear injection $\Theta$ of $W$ into $V$. Particularly, if $\Gamma(W, g)$ is semilinearly imbedded in $\Gamma(V, f)$ with a linear injection $\Theta$, we say simply that $\Gamma(W, g)$ is linearly imbedded in $\Gamma(V, f)$. Using these terminologies, we get the

6) [1]. p. 23. Prop. 10.

7) We always use two $\Gamma$ 's in this sense. 
following fundamental relation between forms and the corresponding groups.

THeOREM 1. Let $V$ and $W$ be vector spaces over a field $K$ of characteristic $\neq 2$, and let $f$ and $g$ be nondegenerate symmetric bilinear forms on $V$ and $W$ respectively. Then, a group $\Gamma(W, g)$ is semi-linearly (linearly) imbedded in a group $\Gamma(V, f)$ if and only if $f$ semi-similarly (similarly) represents $g: f \stackrel{\circlearrowleft}{\rightarrow} g(f \stackrel{\infty}{\rightarrow} g){ }^{8)}$

Proof. The sufficiency is already verified. Conversely, suppose that $\Gamma(W, g)$ is semi-linearly (linearly) imbedded in $\Gamma(V, f)$. Then, there exists a semi-linear (linear) injection $\Theta$ of $W$ into $V$ such that $\Theta\left(\Gamma\left(\Theta(W), f_{1}\right)\right)=\Gamma(W, g)$, where $f_{1}$ is the restriction of $f$ to $\Theta(W)$. Thus, we are reduced to the following lemma.

Lemma. Let $\Theta$ be a semi-linear (linear) isomorphism between $W$ and $V$ such that $\Theta(\Gamma(V, f))=\Gamma(W, g)$, then it follows that $f \propto s g(f \infty g)$.

Proof of Lemma. Let $\operatorname{dim} V=n$. If $n=1$, there is nothing to say. For $n \gtrsim 2$, we devide the proof into several parts.

Case A. $\quad n \geq 3, K \neq G F(3)$. Since $\Theta(\Gamma(V, f))=\Gamma(W, \Theta f)=\Gamma(W, g)$ and $\Omega(W, \Theta f) \subset O^{+}(W, \Theta f)$, it is sufficient to show that $g=\lambda \cdot \Theta f, \lambda \in K^{*}$, if $\Omega(W, \Theta f) \subset O^{+}(W, g)$. Let $P$ be any non-isotropic plane in the sense of $\Theta f$. Then, we have an orthogonal decomposition $W=P \oplus P^{\oplus f}{ }^{9)}$ Let $e_{1}$, $e_{2}$ be some orthogonal basis of $P$ and let $e_{3}, \ldots, e_{n}$ be some basis of $P^{\oplus f}$. We set $\Theta f\left(e_{1}, e_{1}\right)=c, \Theta f\left(e_{2}, e_{2}\right)=d$. Since $K \neq G F(3)$, there exists $t \in K^{*}$ such that $t^{2}+d / c \neq 0$. By this $t$, set $x=\left(t^{2}-d / c\right) /\left(t^{2}+d / c\right), y=2 t /\left(t^{2}+d / c\right)$. Then, it follows that $y \neq 0$ and $x^{2}+(d / c) y^{2}=1$. Relative to the above basis $e_{i}(i=1$, $\ldots, n)$ of $W$, the matrix

$$
\sigma=\left(\begin{array}{ccccccc}
x & -(d / c) y & & & & \\
y & x & & & & \\
& & 1 & & & \\
& & & \cdot & & \\
& & & & \cdot & \\
& & & & & 1
\end{array}\right) \in O^{+}(W, \Theta f)
$$

8) This is a generalization of Lemma 1 in [0].

9) $P \Theta f$ means the conjugate space of $P$ relative to $\otimes f$. 
and so

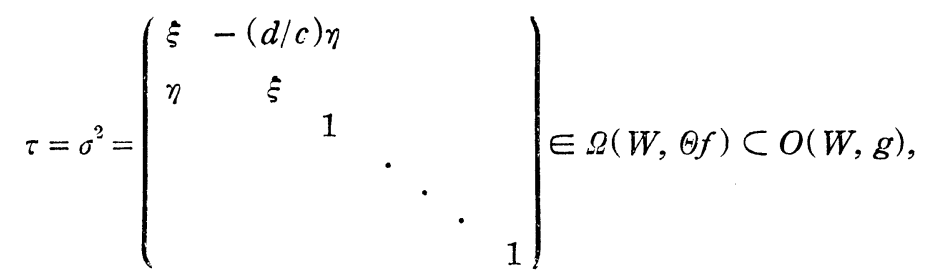

where $\xi=x^{2}-(d / c) y^{2}, \eta=2 x y$. Since $y \neq 0$, it is easily seen that $\xi \neq 1$. Since $\tau g=g$, we have

$$
\begin{aligned}
& g\left(e_{1}, e_{i}\right)=g\left(\tau e_{1}, \tau e_{i}\right)=g\left(\xi e_{1}+\eta e_{2}, e_{i}\right), \\
& g\left(e_{2}, e_{i}\right)=g\left(\tau e_{2}, \tau e_{i}\right)=g\left(-(d / c) \eta e_{1}+\xi e_{2}, e_{i}\right) . \quad(3 \leqq i \leqq n)
\end{aligned}
$$

Therefore

$$
\begin{aligned}
& (\xi-1) g\left(e_{1}, e_{i}\right)+\eta g\left(e_{2}, e_{i}\right)=0, \\
& \quad-(d / c) \eta g\left(e_{1}, e_{i}\right)+(\xi-1) g\left(e_{2}, e_{i}\right)=0 .
\end{aligned}
$$

But the determinant of the coefficients of these linear equations $=(\xi-1)^{2}+(d / c) \eta^{2}$ $=2(1-\xi) \neq 0$. Thus, we get $g\left(e_{1}, e_{i}\right)=g\left(e_{2}, e_{i}\right)=0(i \geq 3)$. This means that $P^{\Theta f} \subset P^{g}$. Comparing the dimensions of the spaces on both sides, we have $P^{\Theta f}=P^{g}$. Now, let $a \neq 0$ be any non-isotropic vector $\in W$ in the sense of $\Theta f$. Since $n \geqslant 3$, there exists non-isotropic planes $P, P^{\prime}$ in the sense of $\Theta f$ (and necessarily in the sense of $g$ ) such that $\langle a\rangle=P \cap P^{\prime}$. Thus, $\langle a\rangle^{\oplus f}=\left(P \cap P^{\prime}\right)^{\Theta f}$ $=P^{\ominus f} \cup P^{\oplus f}=P^{g} \cup P^{\prime g}=\left(P \cap P^{\prime}\right)^{g}=\langle a\rangle^{g}$. Then, by the argument in Lemma 1 in [0], we have $g=\lambda \cdot \Theta f, \lambda \in K^{*}$.

Case B. $n \geqslant 3, K=G F(3)$. It is known that the two finite groups $O^{+}(V, f)$ and $O^{+}(W, g)$ have different orders if $f$ and $g$ are not congruent. ${ }^{10)}$ Therefore if $f$ and $g$ are not semi-similar (similar), there can not be any isomorphism $\Theta$ between $W$ and $V$ such that $\Theta(O(V, f))=O(W, g)$ or $\Theta\left(O^{+}(V, f)\right)=O^{+}(W, g)$. Furthermore, since $n \geqslant 3, \Omega(V, f)$ has index 2 in $O^{+}(V, f),{ }^{11)}$ and so $\Omega(V, f)$ and $\Omega(W, g)$ can not be isomorphic each other if $f$ and $g$ are not semi-similar (similar).

Case C. $n=2$ and $K$ is infinite. We shall show, as in Case A, that $g=\lambda \cdot \Theta f$ if $\Omega(W, \Theta f) \subset O(W, g)$. Let $e_{1}, e_{2}$ be some orthogonal basis relative to

10) [2]. Chapt. VII. $§ 169-170$.

11) [3]. p. 61 . $§ 41$. 
$\Theta f$ in $W$. We may assume without loss of generality that the matrices of the coefficients of $\Theta f$ and $g$ relative to the above basis are of types $\left(\begin{array}{ll}1 & \\ & d\end{array}\right)$ and $\left(\begin{array}{ll}1 & b \\ b & c\end{array}\right)$ respectively. By an element $t \in K^{*}$ such that $t^{2} \pm d \neq 0$, set $x$ $=\left(t^{2}-d\right) /\left(t^{2}+d\right), y=2 t /\left(t^{2}+d\right)$. Then,

$$
\sigma=\left(\begin{array}{cc}
x & -d y \\
y & x
\end{array}\right) \in O^{+}(W, \Theta f)
$$

and

$$
\tau=\sigma^{2}=\left(\begin{array}{cc}
\xi & -d \eta \\
\eta & \xi
\end{array}\right) \in \Omega(W, \Theta f) \subset O(W, g),
$$

where $\xi=x^{2}-d y^{2}, \eta=2 x y$. Comparing the $(1,1)$-components on both sides in the following matric relation

$$
\left(\begin{array}{cc}
\xi & -d \eta \\
\eta & \xi
\end{array}\right)\left(\begin{array}{ll}
1 & b \\
b & c
\end{array}\right)\left(\begin{array}{cc}
\xi & -d \eta \\
\eta & \xi
\end{array}\right)=\left(\begin{array}{ll}
1 & b \\
b & c
\end{array}\right)
$$

we get $\xi^{2}+2 b \xi \eta+c \eta^{2}=1$. Since $\xi^{2}+d \eta^{2}=1$ and $\eta \neq 0$, it follows at once that $2 b \xi+(c-d) \eta=0$. Thus, observing that $K$ contains infinitely many elements, we get $b=0$ and $c=d: g=\Theta f$.

Case D. $n=2, K=G F(q)$ (the finite field with $q$ elements). We shall show that the groups $\Gamma(V, f)$ and $\Gamma(W, g)$ have different orders if $\nu(f)=1$ and $\nu(g)$ $=0$. For $f$ with $\nu(f)=1$, there is a basis of $V$ such that the matrix of the coefficients of $f$ is of type $\left(\begin{array}{ll}0 & 1 \\ 1 & 0\end{array}\right)$ relative to it. The correspondence $\left(\begin{array}{ll}x & \\ 1 / x\end{array}\right)$ $\leftrightarrow x$ gives the isomorphism $O^{+}(V, f) \cong K^{*}$. Thus, we see that the order of $O^{+}(V, f)$ is $q-1$. On the other hand, for $g$ with $\nu(g)=0$, let $\left(\begin{array}{ll}1 & \\ & d\end{array}\right)$ be the matrix of the coefficients of $g$ relative to some orthogonal basis in $W$. Then, the correspondence $\left(\begin{array}{cc}x & -d y \\ y & x\end{array}\right) \leftrightarrow x+\sqrt{-d y}$, with $x^{2}+d y^{2}=1$, gives the isomorphism $O^{+}(W, g) \cong N$, where $N$ is the group composed of all elements $\in L$ $=K(\sqrt{-d})$ with norm $=1$ (relative to this quadratic extension). By Hilbert's lemma, we have $N \cong L^{*} / K^{*}$. Thus, the order of $O^{+}(W, g)$ is $q+1$. Therefore our assertion is true for $\Gamma=O$ or $\Gamma=O^{+}$. Now, by the above correspondence, we have $K^{*} / K^{* 2} \cong O^{+}(V, f) / \Omega(V, f)$. Hence, the order of $\Omega(V, f)$ is $(q-1) / 2$. On the other hand, there exists a homomorphism of $N / N^{2}$ onto $O^{+}(W, g) / \Omega(W, g)$. Since $N$ is the cyclic group of an even order, it follows that $\left[O^{+}(W, g): \Omega(W, g)\right]$ $\leqq 2$. Therefore, order of $\Omega(W, g) \geqslant\left(\right.$ order of $\left.O^{+}(W, g)\right) / 2=(q+1) / 2>(q-1) / 2$ 
$=$ order of $\Omega(V, f)$. Thus, the assertion is also true for $I=\Omega$. Q.E.D.

Remark. We say that $\Gamma(W, \mathrm{~g})$ is abstractly (topologically) imbedded in $\Gamma(V, f)$ if there exists a non-isotropic subspace $W^{\prime} \subset U$ such that $\operatorname{dim} W^{\prime}$ $=\operatorname{dim} W$ and $\Gamma(W, g)$ is isomorphic with $\Gamma\left(W^{\prime}, f^{\prime}\right)$, where $f^{\prime}$ is the restriction of $f$ to $W^{\prime}$, as abstract (topological) groups (when the basic field $K$ is topologized).

We shall close this section with some remarks on the discriminants and the Clifford algebras of forms. ${ }^{12)} \quad$ As for the discriminant $d(f)$ of a form $f$ on $V$, we set

$$
\Delta(f)=(-1)^{n(n-1) / 2} d(f) \text {, where } n=\operatorname{dim} V .
$$

Now, let $g$ be another form on $W$, and let $\S(f)$ and $\S(g)$ be the Clifford algebras of the forms. We put

$$
\begin{aligned}
& \mathfrak{H}(f, g)=\mathfrak{S}(f) \otimes \mathfrak{S}(g) \otimes(d(g), d(f) d(g)), \\
& \mathfrak{B}(f, g)=\mathfrak{H}(f, g) \otimes(-1, d(f) d(g)) .
\end{aligned}
$$

The following properties of these algebras will be used later.

(1) If $n-m \equiv 2(\bmod .4)$, then $\Delta(f) \Delta(g)=-d(f) d(g)$, where $n=\operatorname{dim} V$, $m=\operatorname{dim} W$.

(2) If $n$ is even, $\mathfrak{Y}(a f, g) \sim(a, \Delta(f)) \otimes \mathfrak{U}(f, g), a \in K^{*}$.

If $n$ is odd, $\mathfrak{H}(a f, g) \sim\left(a,(-1)^{(n+1) / 2} d(g)\right) \otimes \mathfrak{U}(f, g)$.

(3) If $n$ is even, $\mathfrak{B}(a f, g) \sim(a, d(f)) \otimes \mathfrak{B}(f, g)$.

If $n$ is odd, $\mathfrak{B}(a f, g) \sim\left(a,(-1)^{(n-1) / 2} d(g)\right) \otimes \mathfrak{B}(f, g)$.

\section{$\S 2$. Local considerations}

In this $\S$, we assume that $K$ is a locally compactly valued field of characteristic $\neq 2$. Let $V$ be an $n$-dimensional vector space over $K$ and let $f$ be a nondegenerate symmetric bilinear form on $V$. If $K$ is the complex number field, then always $\nu(f)=[n / 2]$. Let $g$ be another form on an $m$-dimensional space $W$. Since every form $f$ is congruent to the unit form, $f$ represents $g$ provided $n \geq m$. If $K$ is the real number field, then we get $\nu(f)=\min \left(\iota(f), \iota^{*}(f)\right)$, where $\ell(f)$ is the number of positive coefficients in a canonical form of $f$ and ** $(f)=n-\iota(f)$.

Proposition 3. Let $\dot{K}$ be the real number field and let $f$ and $\mathrm{g}$ be forms

12) [0]. $\S 1$. 
on the spaces $V$ and $W$ respectively. Then, $f$ represents $g: f \rightarrow g$ if and only if $0 \leqq \iota(f)-\iota(g) \leqq n-m\left(0 \leqq \iota^{*}(f)-\iota^{*}(g) \leqq n-m\right)$, where $n=\operatorname{dim} V, m=\operatorname{dim} W$, and $f$ similarly represents $g: f \stackrel{\infty}{\rightarrow}$ if and only if $0 \leqq \nu(f)-\nu(g) \leqq n-m .^{13}$

Proof. By Proposition 1, $f \widetilde{\rightarrow} g$ if and only if there exists a form $h$ such that $f \sim g+h$, namely $\iota(f)=\iota(g+h)=\iota(g)+\iota(h)$. Thus, our first statement is proved. The necessity assertion in the second statement is already proved (Proposition 2). Suppose that $0 \leqq \nu(f)-\nu(g) \leqq n-m$. Then, by suitable $\varepsilon, \eta$ $= \pm 1$, we have $\nu(f)=\ell(\varepsilon f)$ and $\nu(g)=\ell(\eta g)$ and so we are reduced to the first case.

For our purpose in $\S 3$, we give here some formulas on the forms over the real number field. It is easily verified that $d(f) \sim(-1)^{\iota *(f)}$ and $§(f) \sim(-1$, $\left.(-1)^{\iota *(f)\left(c^{*}(f)+1\right) / 2}\right)$. By simple computations, we get the following formulas:

(4) $\mathfrak{X}(f, g) \sim\left(-1,(-1)^{(\iota *(f)-\iota *(g))(\iota *(f)-\iota *(g)+1) / 2}\right)$,

(5) $\mathfrak{B}(f, g) \sim\left(-1,(-1)^{\left(\iota *(f)-\iota^{*}(g)\right)\left(\iota *(f)-\iota^{*}(g)-1\right) / 2}\right)$.

From (4) and (5) it follows that

(6) $\mathfrak{H}(f, g) \sim 1 \quad$ if $\iota^{*}(f)-\iota^{*}(g) \equiv 0,3 \quad(\bmod .4)$, $\mathfrak{A}(f, g)+1 \quad$ if $\iota^{*}(f)-\iota^{*}(g) \equiv 1,2 \quad(\bmod .4)$,

(7) $\mathfrak{B}(f, g) \sim 1 \quad$ if $\iota^{*}(f)-\iota^{*}(g) \equiv 0,1 \quad(\bmod .4)$, $\mathfrak{B}(f, g)+1 \quad$ if $\iota^{*}(f)-\iota^{*}(g) \equiv 2,3 \quad$ (mod. 4$)$.

Now, let $K$ be non-archimedean. We prove some propositions on the local representabilities of the forms.

Proposition 4. Let $K$ be non-archimedean and let $V$ and $W$ be $n$ and $m$ dimensional vector spaces over $K$. Let $f$ and $g$ be forms on $V$ and $W$ respectively. Then,

if $n-m=1, f \Im g$ if and only if $\mathfrak{B}(f, g) \sim 1$,

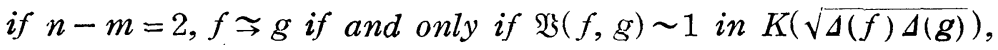

if $n-m \geqslant 3, f \simeq g$ for any $f$ on $V$ and $g$ on $W$.

Proof. By Proposition $1, f \widetilde{\rightarrow} g$ means that there exists a form $h$ such that $f \sim g+h$ and this is equivalent to say that there exists such a form $h$ on an $(n-m)$-dimensional space as $d(f) \sim d(g) d(h)$ and $\S(f) \sim \S(g+h) \sim \S(g)$

13) This condition is trivially satisfied for forms on $V$ and $W(n \geqq m)$ over the complex number field. 
$\otimes((h) \otimes(d(g), d(h))$, namely $d(h) \sim d(f) d(g)$ and $§(h) \sim \S(f) \otimes \circledast(g) \otimes(d(g)$, $d(f) d(g)) \sim \mathfrak{Y}(f, g)$. From the theorem in $[4]^{14)}$ our proposition follows by observing that $\Delta(f) \Delta(g)=-d(f) d(g)$ if $n-m=2$ and $\mathfrak{B}(f, g)=\mathfrak{A}(f, g) \otimes(-1$, $d(f) d(g)$.

Proposition 5. Let $K, V, W, f$ and $g$ be as described in Proposition 4. Then,

i) if $n-m=1, n:$ odd, $f \stackrel{\infty}{\rightarrow} g$ if and only if $\mathfrak{B}(f, g) \sim 1$ in $K(\sqrt{\Delta(g)})$,

ii ) if $n-m=1, n:$ even, $f \stackrel{\infty}{\rightarrow} g$ if and only if $\mathfrak{B}(f, g) \sim 1$ in $K(\sqrt{\Delta(f)})$,

iii ) if $n-m=2, n:$ odd, $f \stackrel{\infty}{\rightarrow} g$ always,

iv) if $n-m=2, n:$ even, $f \stackrel{\infty}{\rightarrow} g$ if and only if $\mathfrak{B}(f, g) \sim 1$

v) if $n-m \geqslant 3, f \stackrel{\infty}{\rightarrow} g$ always. in $K(\sqrt{\Delta(f)}, \sqrt{\Delta(g)})$,

Proof. v) follows at once from Proposition 4. For $n-m \leqq 2, f \stackrel{\infty}{\rightarrow} g$ if and only if $a f \Im g$ for some $a \in K^{*}$ and this is, by Proposition 4, equivalent to say that $\mathfrak{B}(a f, g) \sim 1$ in $K$ if $n-m=1$ and $\mathfrak{B}(a f, g) \sim 1$ in $K(\sqrt{\Delta(a f) \Delta(g)})$ if $n-m$ $=2$. By the formula (3) in $\S 1, f \stackrel{\infty}{\rightarrow} g$ is equivalent to the existence of $a \in K^{*}$ such that

i) $\mathfrak{B}(f, g) \otimes(a, \Delta(g)) \sim 1$ in $K$,

ii) $\mathfrak{B}(f, g) \otimes(a, \Delta(f)) \sim 1$ in $K$,

iii) $\mathfrak{B}(f, g) \otimes(a,-\Delta(g)) \sim 1$ in $K(\sqrt{a \Delta(f) \Delta(g)})$,

iv) $\mathfrak{B}(f, g) \otimes(a, \Delta(f)) \sim 1$ in $K(\sqrt{\Delta(f) \Delta(g)})$.

As for iii), if we take $a \in K^{*}$ such that $a \Delta(f) \Delta(g)$ is not a square, the condition is satisfied for any $f$ and $g$, since then any algebra class with index at most 2 is $\sim 1$ in any proper quadratic extension. On the other hand, the cases i), ii), iv) are reduced to the following lemma.

Lemma. Let $\mathfrak{A}$ be a central simple algebra over our $K$ with index at most 2 , and let $\alpha, \beta$ be $\in K^{*}$. Then, there exists $a \in K$ such that $(a, \alpha) \sim \mathfrak{A}$ in $K(\sqrt{\beta})$ if and only if $\mathfrak{A} \sim 1$ in $K(\sqrt{\alpha}, \sqrt{\beta})$.

Proof of Lemma. The necessity is trivial. Conversely, suppose first that $\beta$ is not a square or both $\beta$ and $\alpha$ are squares. Then, we may take $a=1$. Next, if $\alpha$ is not a square and $\beta$ is a square, we may take such $a$ that is a norm or

14) [4]. Satz 18. 
not relative to the quadratic extension $K(\sqrt{\alpha}) / K$ according as $\mathfrak{U} \sim 1$ or +1 in K.

As an analogue of Proposition 3 we prove the following

Proposition 6. Let $K, V, W, f$ and $g$ be as described in Proposition 4. Suppose that $n>m$. Then, $f$ similarly represents $g: f \stackrel{\infty}{\rightarrow} g$ if and only if $0 \leqq \nu(f)$ $-\nu(g) \leqq n-m$.

Proof. The necessity assertion is already proved (Proposition 2). To prove the sufficiency, suppose first that $n-m \geqslant 3$ or $n-m=2$ and $n$ is odd, then the statement is trivial by Proposition 5 . Therefore there remains to be discussed the three cases i), ii) and iv) in Proposition 5.

i) $0 \leqq \nu(f)-\nu(g) \leqq 1$ implies that $-1 \leqq \nu^{*}(g)-\nu^{*}(f) \leqq 1$.

From this inequality it follows that $\nu^{*}(g)=2, \nu^{*}(f)=1$ and $\nu^{*}(g)=0$ or $\nu^{*}(f)$ $=3$ and $\nu^{*}(g)=4$. By Lemma 2 in [0], these three conditions are equivalent to saying that $\Delta(g)$ is not a square or $\Delta(g)$ is a square and $\mathbb{E}(f) \otimes(-1$, $\left.(-1)^{\left(n^{2}-1\right) / 8} d(f)^{(n+1) / 2}\right) \sim\left(5 .(g) \otimes\left(-1,(-1)^{\left(m^{2}+2 m\right) / 8}\right)\right.$. Thus, these conditions are summed up as $\mathcal{B}(f, g) \sim 1$ in $K(\sqrt{\Delta(g)})$, observing that $\left(-1,(-1)^{\left(n^{2}-1\right) / 8} d(f)^{(n+1) / 2}\right)$ $\otimes\left(-1,(-1)^{\left(m^{2}+2 m\right) / 8}\right) \sim\left(d(f),-(-1)^{m / 2}\right) \sim(d(f),-d(g))$ if $\Delta(g)$ is a square. Then our statement follows from Proposition 5.

ii) is proved in a similar way as in i).

iv) $0 \leqq \nu(f)-\nu(g) \leqq 2$ implies that $-2 \leqq \nu^{*}(g)-\nu^{*}(f) \leqq 2$.

It follows from this that $\nu^{*}(f)=2$ or $\nu^{*}(g)=2$ or $\nu^{*}(f)=\nu^{*}(g)=0$ or 4 . Again by the Lemma 2 in [0], we have $\Delta(f)$ is not a square or $\Delta(g)$ is not a square or $\Delta(f)$ and $\Delta(g)$ are both squares and $\left(5,(f) \otimes\left(-1,(-1)^{\left(n^{2}+2 n\right) / 8}\right) \sim \mathbb{E}(g) \otimes(-1\right.$, $\left.(-1)^{\left(m^{2}+2 m\right) / 8}\right)$. Observing, in the last case, that $\left(-1,(-1)^{\left(n^{2}+2 n\right) / 8} \otimes(-1\right.$, $\left.(-1)^{\left(m^{2}+2 m\right) / 8}\right) \sim\left((-1)^{n / 2},-(-1)^{m / 2}\right) \sim(d(f),-d(g))$, we have $\mathfrak{B}(f, g) \sim 1$ in $K(\sqrt{\Delta(f)}, \sqrt{\Delta(g)})$. Thus, we are reduced to Proposition 5 again.

Now, thus obtained local considerations, containing the case where $n=m$, ${ }^{15}$ are summarized in the following

THEOREM 2. Let $K$ be a locally compactly valued field of characteristic $\neq 2$, and let $V$ and $W$ be vector spaces over $K$ such that $\operatorname{dim} V \geqslant \operatorname{dim} W$. For the nondegenerate symmetric bilinear forms $f, g$ on $V, W$ respectively, the following

15) [0]. Lemma 3. 
three conditions are equivalent.

i) $f$ similarly represents $g: f \stackrel{\infty}{\rightarrow} g$.

ii) $0 \leqq \nu(f)-\nu(g) \leqq \operatorname{dim} V-\operatorname{dim} W$.

(For the case where $\operatorname{dim} V=\operatorname{dim} W$ is even and $K$ is non-archimedean, we add the condition $d(f) \sim d(g)$ ).

iii) $\Gamma(W, g)$ is linearly imbedded in $\Gamma(V, f)$.

Remark. If $O(W, g)$ is imbedded in $O(V, f)$ topologically, we may take a non-isotropic subspace $W^{\prime}$ of $V$ such that $O(W, g) \cong O\left(W^{\prime}, f^{\prime}\right)$ (homeomorphically), where $f^{\prime}$ is the restriction of $f$ to $W^{\prime}$. Thus, we know that $\nu(g)=\nu\left(f^{\prime}\right){ }^{16)}$ Since $f \cong f^{\prime}$, we get $0 \leqq \nu(f)-\nu\left(f^{\prime}\right) \leqq \operatorname{dim} V-\operatorname{dim} W^{\prime}$ by Proposition 2. Therefore, we have $0 \leqq \nu(f)-\nu(g) \leqq \operatorname{dim} V-\operatorname{dim} W$. Furthermore, this inequality comes from the abstract imbedding of the orthogonal groups provided $\operatorname{dim} W$ $\geq 3{ }^{17)}$ For $\operatorname{dim} W \leqq 3$, in order to have that inequality it is sufficient that there exists a homeomorphism as topological spaces between two groups $O(W, g)$ and $O\left(W^{\prime}, f^{\prime}\right){ }^{18)}$

\section{$\S 3$. Hasse principle}

In this $\S$, we assume that $K$ is either a field of algebraic numbers or a field of algebraic functions of one variable over a finite field of characteristic $\neq 2$. Let $K_{\mathfrak{p}}$ be the $\mathfrak{p}$-adic completion of $K$ with respect to a place $\mathfrak{p}$ in $K$. We denote by $V_{\mathfrak{p}}$ the scalar extension of a space $V$ with respect to $K_{\mathfrak{p}}$. First we prove, for the completeness sake, the following well known theorem on the representability of forms.

THEOREM 3. Let $K$ be a field of algebraic numbers or a field of algebraic functions of one variable over a finite field of characteristic $\neq 2$, and let $V$ and $W$ be the vector spaces over $K$ with $\operatorname{dim} V=n$, $\operatorname{dim} W=m$. Let $f$ and $g$ be nondegenerate symmetric bilinear forms on $V$ and $W$ respectively. Then $f$ represents $g: f \widetilde{g} g$ in $K$ if and only if $f \Im g$ in $K_{\mathfrak{p}}$ for every place $\mathfrak{p}$ in $K$.

Proof. The necessity is trivial. We prove the sufficiency separately for several cases.

16) [0]. Lemma 4.

17) [0]. Addendum.

18) For such case, we say that $O(W, g)$ is set-theoretically imbedded in $O(V, f)$. 
i) $n=m$ : We find the proof in [4] (Satz 20).

ii) $\quad n-m=1$ : By the assumption, we have $\mathfrak{B}(f, g) \sim 1$ in $K_{\mathfrak{p}}$ for every finite place $p$ (Proposition 4 ), and $0 \leqq \iota_{p}^{*}(f)-\epsilon_{p}^{*}(g) \leqq 1$ for every real infinite place p. From the formula (7) in $\S 2$, it follows that $\mathfrak{B}(f, g) \sim 1$ in $K_{\mathfrak{p}}$ for every real place $p$. Let $h$ be a unitary form with discriminant $d(f) d(g)$. Then, for finite places $\mathfrak{p}$, we have $(\mathfrak{S}(g+h) \sim \mathfrak{S}(g) \otimes \mathfrak{G}(h) \otimes(d(g), d(f) d(g))$

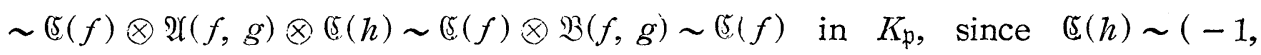
$d(f) d(g))$. On the other hand, for real places $\mathfrak{p}, d(h) \sim(-1)^{\iota_{\mathfrak{p}}^{*}(f)-\iota_{\mathfrak{p}}^{*}(g)}$. Therefore it follows at once that $\iota_{\mathfrak{p}}(f)=\iota_{\mathfrak{p}}(g)+\iota_{\mathfrak{p}}(h)=\iota_{\mathfrak{p}}(g+h)$. Thus $f \sim g+h$ in $K_{\mathfrak{p}}$ for every place $\mathfrak{p}$ in $K$, which reduces the proof to the case where $n=m$.

iii) $n-m=2$. From the assumption, we have $\mathfrak{B}(f, g) \sim 1$ in $K_{\mathfrak{p}}(\sqrt{\Delta(f) \Delta(g)})$ for every finite place $\mathfrak{p}$ (Proposition 4 ) and $0 \leqq \mathfrak{t}_{\mathfrak{p}}^{*}(f)-\mathfrak{t}_{\mathfrak{p}}^{*}(g) \leqq 2$ for every real place $\mathfrak{p}$. If $\iota_{\mathfrak{p}}^{*}(f)-\mathfrak{c}_{\mathfrak{p}}^{*}(g)=0$ or 1 for a real place $\mathfrak{p}$, then $\mathfrak{B}(f, g) \sim 1$ in $K_{\mathfrak{p}}$ and necessarily in $K_{\mathfrak{p}}(\sqrt{\Delta(f) \Delta(g)})$ by the formula (7). While, if $\iota_{\mathfrak{p}}^{*}(f)-\mathfrak{c}_{\mathfrak{p}}^{*}(g)=2$, then $\Delta(f) \Delta(g)=-d(f) d(g) \quad((1)$ in $\S 1)$ is negative in $K_{\mathfrak{p}}$ and so $\mathfrak{B}(f, g) \sim 1$ in $K_{\mathfrak{p}}(\sqrt{\Delta(f) \Delta(g)})$ for such real place $\mathfrak{p}$. Therefore from the fundamental statement on the splitting of algebras, we get $\mathfrak{B}(f, g) \sim 1$ in $K(\sqrt{\Delta(f) \Delta(g)})$ $=K(\sqrt{-d(f) d(g)})$. Now, set $d=d(f) d(g), \widetilde{\varsigma}=(s(f) \otimes(\mathfrak{s}(g) \otimes(d(g), d)$. Then,

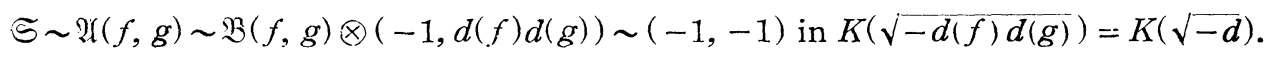
Thus, from the theorem in $[4]^{19)}$ there exists a binary form $h$ over $K$ such that

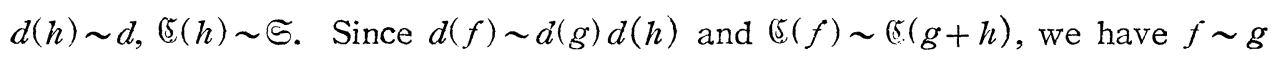
$+h$ in $K_{\mathfrak{p}}$ for every finite place $\mathfrak{p}$. As to a real place $\mathfrak{p}, d(h) \sim d(f) d(g) \mathrm{im}$ plies that $\iota_{\mathfrak{p}}^{*}(f) \equiv \iota_{\mathfrak{p}}^{*}(g)+\iota_{\mathfrak{p}}^{*}(h)(\bmod .2)$. We have $\left(5(h) \sim \mathfrak{H}(f, g) \sim 1\right.$ if $\iota_{\mathfrak{p}}^{*}(f)$ - $\iota_{p}^{*}(g) \equiv 0,3$ (mod. 4$)$ and $\left((h)+1\right.$ if $\iota_{p}^{*}(f)-\varsigma_{p}^{*}(g) \equiv 1,2$ (mod. 4) ((6) in $\left.\S 2\right)$. Since $\Subset(h) \sim\left(-1,(-1)^{\iota_{\mathfrak{p}}^{*}(h)\left(\epsilon_{\mathfrak{p}}^{*}(h)+1\right) / 2}\right)$, it follows at once that $\iota_{\mathfrak{p}}^{*}(f)=\mathfrak{q}_{\mathfrak{p}}^{*}(g)$ $+\iota_{\mathfrak{p}}^{*}(h)=\iota_{\mathfrak{p}}^{*}(g+h)$. Thus, we get $f \sim g+h$ in $K_{\mathfrak{p}}$ for all places $\mathfrak{p}$ in $K$, and we are reduced to the case where $n=m$.

iv) $n-m=3$. Let $d=d(f) d(g)$ and $\subseteq=(S(f) \otimes(s(g) \otimes(d(g), d)$, then there exists a ternary form $h$ such that $d(h) \sim d$ and $\mathbb{E}(h) \sim \Im^{20)}$ Therefore $d(f)$ $\sim d(g) \cdot d(h)$ and $\mathbb{E}(f) \sim \mathbb{S}(g+h)$ and so $f \sim g+h$ in $K_{\mathfrak{p}}$ for every finite place p. As to a real place $\mathfrak{p}$, the similar consideration as in iii) shows that $\mathfrak{w}^{*}(f)$ $=\iota_{p}^{*}(g+h)$. Thus, we are also reduced to the case $n=m$.

19) [4]. Satz 18.

20) [4]. Satz 18. 
v) $n-m \geqslant 4$. Let $d=d(f) d(g), \Im=\Subset(f) \otimes\left((g) \otimes(d(g), d)\right.$ and $\iota_{\mathfrak{p}}^{*}=\iota_{\mathfrak{p}}^{*}(f)$ $-\iota_{\mathfrak{p}}^{*}(g)$, where $\mathfrak{p}$ is any real place in $K$. Then, we get $d \sim(-1)^{* *}$ and $\subseteq \sim \mathfrak{H}(f, g)$ $\sim\left(-1,(-1)^{\left(\iota_{\mathfrak{p}}^{*}(f)-\iota_{\mathfrak{p}}^{*}(g)\right)\left(\iota_{\mathfrak{p}}^{*}(f)-\iota_{\mathfrak{p}}^{*}(g)+1\right) / 2}\right)=\left(-1,(-1)^{\iota_{\mathfrak{p}}^{*}\left(\iota_{\mathfrak{p}}^{*}+1\right) / 2}\right)$. Therefore, there exists a form $h$ wtih $d \sim d(h), \subseteq \sim\left(\mathfrak{S}(h)\right.$ and $\mathfrak{k}=\iota_{\mathfrak{p}}(h)$ for every real place $\mathfrak{p}^{21)}$ Thus, we get $f \sim g+h$ in $K_{\mathfrak{p}}$ for every place $\mathfrak{p}$ in $K$ and we are reduced to the case $n=m$. Q.E.D.

Next, we prove the following theorem of Hasse type on the similar representability of forms.

Theorem 4. Let $K, V, W, f$ and $g$ be as described in Theorem 3. Then, $f \stackrel{\infty}{\rightarrow} g$ in $K$ if and only if $f \stackrel{\infty}{\rightarrow} g$ in $K_{\mathfrak{p}}$ for every place $\mathfrak{p}$ in $K$.

Proof. The necessity is trivial. Conversely, if $n=m$, the sufficiency is proved in [0], and if $n-m \geqslant 3$ there is nothing to say since then any $f$ represents any $g$. Therefore we shall prove the sufficiency in the following four cases.

i) $n-m=1, n$ : odd. By the assumption, we have $0 \leqq \nu_{\mathfrak{p}}(f)-\nu_{\mathfrak{p}}(g) \leqq 1$ for any real place $\mathfrak{p}$. Multiplying $\varepsilon, \eta \in K$ with suitable positive or negative signs relative to real places on $f$ and $g$ respectively, we may assume that $\nu_{\mathfrak{p}}(f)$ $=\iota_{\mathfrak{p}}^{*}(f)$ and $\nu_{\mathfrak{p}}(g)=\mathfrak{c}_{\mathfrak{p}}^{*}(g)$ for every real place $\mathfrak{p}$. For a finite place $\mathfrak{p}$, we have $\mathfrak{B}(f, g) \sim 1$ in $K_{\mathfrak{p}}(\sqrt{\Delta(g)})$ from Proposition 5 . On the other hand, for a real place $\mathfrak{p}$, we have $\mathfrak{B}(f, g) \sim 1$ in $K_{\mathfrak{p}}$ from $(7)$ in $\S 2$. Thus, $\mathfrak{B}(f, g) \sim 1$ in $K_{\mathfrak{p}}(\sqrt{\Delta(g)})$ for every place $\mathfrak{p}$ in $K$, and so $\mathfrak{B}(f, g) \sim 1$ in $K(\sqrt{\Delta(g)})$ and $\mathfrak{B}(f, g)$ $\sim(a, \Delta(g))$ in $K$ with some $a \in K^{*}$. Next, we replace $a$ by a totally positive element $\in K^{*}$. If $\Delta(g)$ is a square in $K$, then we may put $a=1$. If $\Delta(g)$ is not a square, let $L=K(\sqrt{\Delta(g)})$ and let $\mathfrak{M}$ be the totality of real places $\mathfrak{p}$ such that $\Delta(g)$ is positive in $K_{p}$, then by the lemma in $[0],{ }^{23)}$ there exists an element $c \in L$ such that $a N_{L / K} c$ is positive in $K_{\mathfrak{p}}$ for all $\mathfrak{p} \in \mathfrak{M}$. Set $b=a N_{L / K} c$, then $\mathfrak{B}(f, g) \sim(a, \Delta(g)) \sim(b, \Delta(g))$ in $K$. For all real infinite places $p$ such that $\mathfrak{p} \notin \mathfrak{M}, b$ must be positive in $K_{\mathfrak{p}}$, since $1 \sim \mathfrak{B}(f, g) \sim(\breve{b}, \Delta(g))$ and $\Delta(g)$ is negative in such $K_{\mathrm{p}}$. Thus, $b$ is totally positive and $\mathfrak{B}(b f, g) \sim \mathfrak{B}(f, g) \otimes(b, \Delta(g)) \sim 1$ in $K$. Therefore, $b f \widetilde{\rightarrow} g$ in $K_{\mathfrak{p}}$, since $\iota_{\mathfrak{p}}^{*}(b f)=\iota_{\mathfrak{p}}^{*}(f)$ for any real place $\mathfrak{p}$. Thus, we are reduced to Theorem 3 .

21) [4]. Satz 21.

22) [0]. Theorem 1 .

23) [0]. Lemma 5 . 
ii) $n-m=1, n$ : even. This case is treated by the similar method as in i).

iii) $n-m=2, n$ : odd. To prove this case we need the following

Lemma. Let $\mathfrak{R}$ be any finite set of finite places in $K$, then there exists an element $a \in K$ such that $a$ is not a square in $K_{\mathfrak{p}}$ for every place $\mathfrak{p}$ in $\mathfrak{R}$ and $a$ has an arbitrarily given (positive or negative) sign in each $K_{\mathfrak{p}}$ for every real place $\mathfrak{p}$ when $K$ is a number field.

Proof of lemma. Let $\mathfrak{N}^{\prime}$ be a set of finite places in $K$ containing places of an even number such that $\mathfrak{N}^{\prime} \supset \mathfrak{N}$. Then, there exists a cyclic algebra $\mathfrak{U}=(b$, $K(\sqrt{\beta}), \sigma)$ with $\mathfrak{p}$-invariant $\left(\frac{\mathfrak{A}}{\mathfrak{p}}\right) \equiv \frac{1}{2}(\bmod .1)$ for every place $\mathfrak{p} \in \mathfrak{N}^{\prime}$ and $\left(\frac{\mathfrak{A}}{\mathfrak{p}}\right)$ $\equiv 0$ (mod. 1 ) for every $\mathfrak{p} \notin \mathfrak{R}^{\prime}$. Thus, $b$ is not a square in $K_{\mathfrak{p}}$ for $\mathfrak{p} \in \mathfrak{N}^{\prime}$. Since $c \in K$ is a square in $K_{\mathfrak{p}}$ if and only if $c$ is a square mod. $p^{e p}$ with a suitable positive integer $e_{\mathfrak{p}}$, the element $a \in K$ which satisfies the equation $x \equiv b$ $\bmod \underset{\mathfrak{p} \in \mathfrak{N}^{\prime}}{\prod^{e} \mathfrak{p}}$ and the given sign condition is the desired one.

Proof of iii). Similarly as in i) we may assume that $0 \leqq \iota_{\mathfrak{p}}^{*}(f)-\iota_{\mathfrak{p}}^{*}(g) \leqq 2$ for every real place $\mathfrak{p}$. If $\iota_{\mathfrak{p}}^{*}(f)-\iota_{\mathfrak{p}}^{*}(g)=0,1$ then $\mathfrak{B}(f, g) \sim 1$ in $K_{\mathfrak{p}}((7)$ in $\S 2)$. Now, let $\varepsilon$ be an element $\in K$ such that $\varepsilon$ is positive in $K_{\mathfrak{p}}$ for $\mathfrak{p}$ with $\iota_{\mathfrak{p}}^{*}(f)-\iota_{\mathfrak{p}}^{*}(g)=0,1$ and is negative in $K_{\mathfrak{p}}$ for $\mathfrak{p}$ with $\iota_{\mathfrak{p}}^{*}(f)-\iota_{\mathfrak{p}}^{*}(g)=2$. Then, for the latter $\mathfrak{p}$, we have $\iota_{\mathfrak{p}}^{*}(\varepsilon f)=\iota_{\mathfrak{p}}^{*}(\varepsilon g)$ since $n-m=2$ and $\iota_{\mathfrak{p}}^{*}(\varepsilon f)=n-\iota_{\mathfrak{p}}^{*}(f)$, $\iota_{\mathfrak{p}}^{*}(\varepsilon g)=m-\iota_{\mathfrak{p}}^{*}(g)$. Thus, we may assume that $\iota_{\mathfrak{p}}^{*}(f)-\iota_{\mathfrak{p}}^{*}(g)=0,1$ for all real places $\mathfrak{p}$ and so $\mathfrak{B}(f, g) \sim 1$ in $K_{\mathfrak{p}}$ for all real places $\mathfrak{p}$. Our statement is proved if we can show that there exists a totally positive $a \in K^{*}$ such that $\mathfrak{B}(a f, g)$ $\sim \mathfrak{B}(f, g) \otimes(a,-\Delta(g)) \sim 1$ in $K_{\mathfrak{p}}(\sqrt{a \Delta(f) \Delta(g)})$ for every finite place $\mathfrak{p}$ (Proposition 4). To do this, suppose first that $-\Delta(g)$ is a square in $K$. Let $\mathfrak{R}$ be the totality of all finite places $\mathfrak{p}$ such that $\mathfrak{B}(f, g)+1$ in $K_{\mathfrak{p}}$. Then, from the above lemma, there exists $b \in K$ such that $a=-b \Delta(f)$ is totally positive in $K$ and $b$ is not a square in $K_{\mathfrak{p}}$ for $\mathfrak{p} \in \mathfrak{\Re}$. Thus, if $\mathfrak{p} \notin \Re$, then $\mathfrak{B}(f, g) \sim 1$ in $K_{\mathfrak{p}}$ and if $\mathfrak{p} \in \mathfrak{N}$, then $\mathfrak{B}(f, g) \sim 1$ in $K_{\mathfrak{p}}(\sqrt{-a \Delta(f)})$ since $K_{\mathfrak{p}}(\sqrt{-a \Delta(f)})$ is a proper quadratic extension. Therefore we get $\mathfrak{B}(f, g) \sim 1$ in $K(\sqrt{-a \Delta(f)})$ $=K(\sqrt{a \Delta(f) \Delta(g)})$. Next, suppose that $-\Delta(g)$ is not a square in $K$. Set $\mathfrak{B}^{*}$ $=\mathfrak{B}(f, g) \otimes(\Delta(f),-\Delta(g))$. If $\mathfrak{B}^{*} \sim 1$ in $K$, then we take an element $c \in K$ such that $a=c \Delta(f) \Delta(g)$ is totally positive in $K$. It follows that $\mathfrak{B}(f, g)$ $\otimes(a,-\Delta(g)) \sim \mathcal{B}(f, g) \otimes(c \Delta(f) \Delta(g),-\Delta(g)) \sim \mathfrak{B}(f, g) \otimes(\Delta(f),-\Delta(g)) \otimes(c$, $-\Delta(g)) \sim \mathfrak{B}^{*} \otimes(c,-\Delta(g)) \sim 1$ in $K(\sqrt{c})=K(\sqrt{a \Delta(f) \Delta(g)})$. On the other hand, 
if $\mathfrak{B}^{*}+1$ in $K$, then $\mathfrak{B}^{*} \sim(\alpha, \beta), \alpha, \beta \in K^{*}$. For a real place $\mathfrak{p}$, we have $\mathfrak{B}^{*}$ $\sim \mathcal{B}(f, g) \otimes\left((-1)^{(n-1) / 2}(-1)^{\ell_{\mathfrak{p}}^{*}(f)},(-1)^{(n-1) / 2}(-1)^{\iota_{\mathfrak{p}}^{*}(g)}\right) \sim\left((-1)^{(n-1) / 2}(-1)^{\iota_{\mathfrak{p}}^{*}(f)}\right.$, $\left.(-1)^{(n-1) / 2}(-1)^{\iota_{\mathfrak{p}}^{*}(g)}\right)$. Thus, $\mathfrak{B}^{*} \sim 1$ in $K_{\mathfrak{p}}$ for $\mathfrak{p}$ such that $\iota_{\mathfrak{p}}^{*}(f)-\iota_{\mathfrak{p}}^{*}(g)=1$. Now let $\mathfrak{M}$ be the totality of real infinite places $\mathfrak{p}$ such that $\beta$ is positive in $K_{\mathfrak{p}}$, then there exists an element $c \in L=K(\sqrt{\beta})$ such that $\alpha^{\prime}=\alpha N_{L / K} c$ is positive in $K_{\mathfrak{p}}$ for $\mathfrak{p} \in \mathfrak{M}$. For $\mathfrak{p} \notin \mathfrak{M}$ such that $\mathfrak{B}^{*} \sim 1$ in $K_{\mathfrak{p}}$, we have $\alpha^{\prime}$ is positive in $K_{\mathfrak{p}}$. Therefore we may assume that $\alpha$ is positive in $K_{\mathfrak{p}}$ for $\mathfrak{p}$ such that $\mathfrak{B}^{*} \sim 1$ in $K_{\mathfrak{p}}$. Next, let $\mathfrak{M}^{\prime}$ be the totality of $\mathfrak{p}^{\prime}$ s such that $\alpha$ is positive in $K_{\mathfrak{p}}$, then there exists an element $c^{\prime} \in L^{\prime}=K(\sqrt{\alpha})$ such that $\beta^{\prime}=\beta N_{L^{\prime} / K} c^{\prime}$ has the same sign as $\Delta(f) \Delta(g)$ in $K_{\mathfrak{p}}$ for every $\mathfrak{p} \in \mathfrak{M}^{\prime}$. For $\mathfrak{p} \notin \mathfrak{M}^{\prime}$, since $\alpha$ is negative in $K_{\mathfrak{p}}, \mathfrak{B}^{*}$ $\sim\left((-1)^{(n-1) / 2}(-1)^{\iota_{\mathfrak{p}}^{*}(f)},(-1)^{(n-1) / 2}(-1)^{\iota_{\mathfrak{p}}^{*}(g)}\right)+1$ in $K_{\mathfrak{p}}$, hence $\beta^{\prime}$ is also negative. On the other hand, this relation implies that $\iota_{p}^{*}(f)=\iota_{\mathfrak{p}}^{*}(g)$ and so $\Delta(f) \Delta(g)$ $\sim(-1)^{(n-1) / 2}(-1)^{\iota_{\mathfrak{p}}^{*}(f)},(-1)^{(m-1) / 2}(-1)^{\ell_{\mathfrak{p}}^{*}(g)}=-1$. 'Thus, $\beta^{\prime}$ has again the same sign as $\Delta(f) \Delta(g)$ in $K_{\mathfrak{p}}$ for $\mathfrak{p} \notin \mathfrak{M}^{\prime}$. Put $a=\beta^{\prime} \Delta(f) \Delta(g)$. Then, $a$ is totally positive in $K$ and $\mathfrak{B}(f, g) \otimes(a,-\Delta(g)) \sim \mathfrak{B}(f, g) \otimes\left(\beta^{\prime} \Delta(f) \Delta(g),-\Delta(g)\right)$ $\sim B^{*} \otimes\left(\beta^{\prime},-\Delta(g)\right) \sim\left(\alpha, \beta^{\prime}\right) \otimes\left(\beta^{\prime},-\Delta(g)\right) \sim\left(\beta^{\prime},-\alpha \Delta(g)\right) \sim 1$ in $K\left(\sqrt{\beta^{\prime}}\right)$ $=K(\sqrt{a \Delta(f) \Delta(g)})$.

iv) $n-m=2, n$ : even. As in iii), we may assume that $\mathfrak{B}(f, g) \sim 1$ in $K_{\mathrm{p}}$ for real places $\mathfrak{p}$ and $\mathfrak{B}(f, g) \sim 1$ in $K_{\mathfrak{p}}(\sqrt{\Delta(f)}, \sqrt{\Delta(g)})$ for finite places $\mathfrak{p}$, and we shall show that there exists a totally positive $a \in K$ such that $\mathfrak{B}(f, g) \sim(a, \Delta(f))$ in $K_{\mathfrak{p}}(\sqrt{\Delta(f) \Delta(g)})$ for all finite places $\mathfrak{p}$ (Proposition 4). If one of $\Delta(f)$ or $\Delta(g)$ is a square in $K$, then we may take $a=1$. If $\Delta(f) \sim \Delta(g)+1$ in $K$, then $\mathfrak{B}(f, g)$ $\sim 1$ in $K(\sqrt{\Delta(f)})$ and so $\mathfrak{B}(f, g) \sim(b, \Delta(f))$ in $K$ for some $b \in K$. Then, we may replace $b$ by totally positive $a$, since $\mathfrak{B}(f, g) \sim 1$ in $K_{\mathfrak{p}}$ for any real place $\mathfrak{p}$. Therefore it remains to be considered the case where $L=K(\sqrt{\Delta(f)}, \sqrt{\Delta(g)})$ is a proper biquadratic extension. Now, let $\mathfrak{M}$ be the totality of all finite places $\mathfrak{p}$ such that $\Delta(f) \sim \Delta(g)+1$ in $K_{\mathfrak{p}}$ and let $\mathfrak{M}_{0}$ be the totality of all $\mathfrak{p} \in \mathfrak{M}$ such that $\mathfrak{B}(f, g)+1$ in $K_{\mathfrak{p}}$. Set $\mathfrak{M}_{1}=\mathfrak{M}_{0}$ if $\mathfrak{M}_{0}$ contains an even number of $\mathfrak{p}$ and $\mathfrak{M}_{1}=\mathfrak{M}_{0}+\{\mathfrak{q}\}$, where $\mathfrak{q}$ is a finite place in $K$ such that $\Delta(f)+1$ in $K_{\mathfrak{q}}$ and $\Delta(f)$ $+\Delta(g)$ in $K_{q},{ }^{24)}$ if $\mathfrak{M}_{0}$ contains an odd number of $\mathfrak{p}$. Let $\mathfrak{B}^{*}$ be the algebra such that $\left(\frac{\mathfrak{B}^{*}}{\mathfrak{p}}\right) \equiv \frac{1}{2}(\bmod .1), \mathfrak{p} \in \mathfrak{M}_{1}$ and $\left(\frac{\mathfrak{B}^{*}}{\mathfrak{p}}\right) \equiv 0$ (mod. 1) for all places $\mathfrak{p} \notin \mathfrak{M}_{1}$. Since $\Delta(f)+1$ in $K_{\mathfrak{p}}$ for $\mathfrak{p} \in \mathfrak{M}_{1}$, $\mathfrak{B}^{*} \sim 1$ in $K_{\mathfrak{p}}(\sqrt{\Delta(f)})$ for all places $\mathfrak{p}$ in $K$, thus $\mathfrak{B}^{*} \sim(a, \Delta(f))$, where we may assume that $a$ is totally positive in

24) Since $L / K$ is biquadratic, we may take such $q$. 
$K$ since $\mathfrak{B}^{*} \sim 1$ in $K_{\mathfrak{p}}$ for every real $\mathfrak{p}$. On the other hand, for a finite place $\mathfrak{p}$ with $\Delta(f) \Delta(g)+1$ in $K_{\mathfrak{p}}$, we have $\mathfrak{B}(f, g) \sim \mathfrak{B}^{*} \sim 1$ in $K_{\mathfrak{p}}(\sqrt{\Delta(f) \Delta(g)})$, for a finite place $\mathfrak{p}$ with $\Delta(f) \sim \Delta(g)+1$ in $K_{\mathfrak{p}}$, we have $\mathfrak{B}(f, g)+1$ and $\mathfrak{B}^{*}+1$ in $K_{\mathfrak{p}}$ if $\mathfrak{p} \in \mathfrak{M}_{1}$ and $\mathfrak{B}(f, g) \sim \mathfrak{B}^{*} \sim 1$ if $\mathfrak{p} \notin \mathfrak{M}_{1}$, for a finite place $\mathfrak{p}$ with $\Delta(f)$ $\sim \Delta(g) \sim 1$ in $K_{\mathfrak{p}}$ it follows from the assumption that $\mathfrak{B}(f, g) \sim 1$ in $K_{\mathfrak{p}}$ and $\mathfrak{B}^{*} \sim 1$ in $K_{\mathfrak{p}}$, and lastly for a real places $\mathfrak{p}$, we get $\mathfrak{B}(f, g) \sim \mathfrak{B}^{*} \sim 1$ in $K_{\mathfrak{p}}$. Therefore $\mathfrak{B}(f, g) \sim \mathfrak{B}^{*}$ in $K_{\mathfrak{p}}(\sqrt{\Delta(f) \Delta(g)})$ for all $\mathfrak{p}$. Thus, $\mathfrak{B}(f, g) \sim \mathfrak{B}^{*}$ $\sim(a, \Delta(f))$ in $K_{\mathfrak{p}}(\sqrt{\Delta(f) \Delta(g)})$. Q.E.D.

THEOREM 5. Let $K, V, W, f$ and $g$ be as described in Theorem 3. Then, $f$ similarly represents $g: f \stackrel{\infty}{\rightarrow} g$ in $K$ if and only if $0 \leqq \nu_{p}(f)-\nu p(g) \leqq \operatorname{dim} V$ - $\operatorname{dim} W$ for every plase $\mathfrak{p}$ in $K$.

Proof. The necessity is almost trivial (Proposition 2). Conversely, if $\operatorname{dim} V=\operatorname{dim} W$, then $\nu_{\mathfrak{p}}(f)=\nu_{\mathfrak{p}}(g)$ for all $\mathfrak{p}$ implies that $f \infty g$ in $K_{\mathfrak{p}}$ for all $p$ if $\operatorname{dim} V$ is odd. ${ }^{25)}$ On the other hand, if $\operatorname{dim} V$ is even, we know that $\Delta(f)$ is a square in $K_{\mathfrak{p}}$ if and only if $\Delta(g)$ is so. ${ }^{26)} \quad$ Thus, we get $\Delta(f) \sim \Delta(g)$ (and $d(f)$ $\sim d(g)$ ). Then, we again have $f \infty g$ in $K_{\mathfrak{p}}$ for all $\mathfrak{p}^{27)}$ Therefore $f \infty g$ in $K$. Next, if $\operatorname{dim} V>\operatorname{dim} W$, then we get $f \stackrel{\infty}{\rightarrow} g$ in $K_{\mathfrak{p}}$ for all $\mathfrak{p}$ (Theorem 2) and we are reduced to Theorem 4. Q.E.D.

THEOREM 6. Under the same assumption as in Theorem 3, if $f$ semisimilarly represents $g: f \stackrel{\leftrightarrow}{\rightarrow} g$ in $K_{\mathfrak{p}}$ for every place $\mathfrak{p}$ in $K$, then $f$ similarly represents $g: f \stackrel{\infty}{\rightarrow} g$ in $K$.

Proof. $f \hookrightarrow g$ in $K_{\mathfrak{p}}$ implies that $0 \leqq \nu_{\mathfrak{p}}(f)-\nu_{\mathfrak{p}}(g) \leqq \operatorname{dim} V-\operatorname{dim} W$ (Proposition 2). Thus, our statement comes from Theorem 5 immediately.

Now, we shall transfer the so obtained theorems of Hasse type to that of orthogonal groups.

From Theorem 1 and Theorem 4, we get

THEOREM 7. Under the same assumption as in Theorem 3, a group $I(W, g)$ is linearly imbedded in $\Gamma(V, f)$ if and only if $\Gamma\left(W_{p}, g\right)$ is linearly imbedded in $\Gamma\left(V_{\mathfrak{p}}, f\right)$ for every place $\mathfrak{p}$ in $K$.

From Theorem 1 and Theorem 6, we get

\footnotetext{
25) [0]. Lemma 3.

26) [0]. Lemma 2.

27) See 25).
} 
THEOREM 8. If $\Gamma\left(W_{\mathfrak{p}}, g\right)$ is semi-linearly imbedded in $\Gamma\left(V_{\mathfrak{p}}, f\right)$ for every place $\mathfrak{p}$ in $K$, then $\Gamma(W, g)$ is linearly imbedded in $\Gamma(V, f)$.

From Theorem 5 and Remark in $\S 2$ we have the following theorems.

THEOREM 9. If $O\left(W_{\mathfrak{p}}, g\right)$ is topologically imbedded in $O\left(V_{\mathfrak{p}}, f\right)$ for every place $p$ in $K$, then $O(W, g)$ is linearly imbedded in $O(V, f)$.

TheOREM 10. Suppose that $\operatorname{dim} W \geqslant 3$. If $O\left(W_{\downarrow}, g\right)$ is abstractly imbedded in $O\left(V_{\mathfrak{p}}, f\right)$ for every place $\mathfrak{p}$ in $K$, then $O(W, g)$ is linearly imbedded in $O(V, f)$.

TheOREM 11. Suppose that $\operatorname{dim} W \leqq 3$. If $O\left(W_{\mathfrak{p}}, g\right)$ is set-theoretically imbedded $^{28)}$ in $O\left(V_{\mathfrak{p}}, f\right)$ for every place $\mathfrak{p}$ in $K$, then $O(W, g)$ is linearly imbedded in $O(V, f)$.

\section{REFERENCES}

[0] T. Ono, Arithmetic of Orthogonal Groups, J. Math. Soc. Japan, Vol. 7, No. 1, pp. 79-91, 1955.

[1] J. Dieudonné, Sur les groupes classiques, Actual. Sci. Ind., No. 1040, Paris (Hermann), 1948.

[2] L. E. Dickson, Linear groups, Leipzing (Teubner), 1901.

[3] J. Dieudonné, On the automorphisms of the classical groups, Memoirs of the Amer. Math. Soc., Vol. 2, 1952.

[4] E. Witt, Theorie der quadratischen Formen in beliebigen Körpern, Crelles J., Bd. 176, pp. 31-44, 1937.

Mathematical Institute, Nagoya University

28) See Remark in $\S 2$. 